\title{
A clinical and pathological investigation of perinatal mortality in dairy calves: preliminary results from a recent prospective study
}

J.F. Mee

Teagasc, Fermoy, Ireland

Email:john.mee@teagasc.ie

Introduction Perinatal mortality (calf death within 48 hours of birth following a gestation period of at least 260 days) has increased recently internationally and in Irish dairy herds (Mee et al., 2008). While traditionally the predominant aetiology was dystocia (Mee, 2009) the current causes of such loss are unclear. Results from necropsy examinations of calves at regional veterinary laboratories provide causes of death but may be biased towards causes of death not immediately obvious to the farmer or their veterinarian. Such passive surveillance data may also lack detailed epidemiological background information on the circumstances of the calf death. In contrast, a prospective active surveillance study design can gather both detailed anamnestic and pathological data on all calf mortality, irrespective of the perceived cause of death and hence give a more comprehensive picture of the causes of total herd perinatal mortality.

Materials and methods Between January and May 2009, 40 necropsy examinations were carried out on calves which died within $48 \mathrm{~h}$ of calving on seven spring-calving dairy farms (1-15 calves/farm). In addition, blood samples were collected from a random selection of ten pregnant dams per herd. Epidemiological data (single/twin, parity, sex, breed, body condition score, gestation length, calving date and time and degree of assistance, calf clinical signs and time of death) were collected on carcass submission questionnaire forms and necropsies (body and organ weights, gross pathology, histopathology, microbiology, serology and micronutrient sampling) were carried out at Moorepark Dairy Production Research Centre. Additional laboratory analyses were conducted by the veterinary laboratories of the Department of Agriculture and Food in Cork (microbiological culture, fetal serology for Leptospira hardjo and Neospora caninumImmunocomb test kits), Limerick (maternal blood selenium) and Backweston (liver copper, cobalt and selenium and kidney selenium analysis and BVDv tissue PCR, and BVDv serum antigen and antibody assays), the Veterinary Sciences Division of AFBI in Stormont, Northern Ireland (maternal blood plasma inorganic iodine, copper and magnesium) and the Scottish Agricultural Colleges (SAC), Penicuik, Scotland (neonatal hepatic and serum vitamin A and E analyses).

Results Of the 40 carcasses submitted, 38 (95\%) were singletons, 29 (73\%) were from pluriparae, 28 (70\%) were from Holstein-Friesian sires and 23 (58\%) were male. Sixteen calves (40\%) were unobserved/unassisted at calving and 15 (38\%) had difficult (score $\geq 3 ; 1-5$ scale) calvings. The mean (sd) death weight of the calves was $34.6(8.2) \mathrm{kg}$. The majority of calves were dead at birth/stillborn (78\%) with 5, 10 and $8 \%$ dying $1 \mathrm{~min}-1 \mathrm{~h},>1 \mathrm{~h}-24 \mathrm{~h}$ and $>24-48 \mathrm{~h}$ after calving, respectively. In over half the calves their lungs were atelectatic (53\%) with the remained partially atelectatic (33\%) or inflated (15\%). Results were available for 32 calves where serum was examined for BVD antigen, of which none had antigen and 4 had antibodies ( 3 of which were $>1 \mathrm{~h}$ old), respectively. Of 26 calves with results for $L$ hardjo serum antibodies, 3 had titres $\geq 1: 50$ (all 3 were $>1$ h old). Of 31 calves with results for $N$. caninum antibodies, 2 had inconclusive or positive titres ( 1 of which was $>1$ h old). Of 33 calves with abomasal contents culture results none yielded significant isolates. Mean (sd) thyroid gland weight was $14.1 \mathrm{~g}(5.95)$ with $2.5 \%$ of glands $>30 \mathrm{~g}$ and a mean (sd) thyroid/body weight ratio of $0.04(0.014)$. The mean (sd) liver copper $(n=36)$, selenium (36) and cobalt (11) concentrations were $1.8(0.74)$ $\mathrm{mmol} / \mathrm{kg}, 6.7$ (2.19) umol/kg and $0.2(0.07) \mathrm{umol} / \mathrm{kg}$, WM, respectively. The mean (sd) kidney selenium ( $\mathrm{n}=35$ ) concentration was 9.1 (1.69) umol/kg. Using the analyzing laboratories reference ranges (liver Co $0.7-5 \mathrm{umol} / \mathrm{L}$, liver $\mathrm{Cu}$ 0.06-2.5 mmol/L, liver Se 0.75-3 umol/L and kidney Se 5-20 umol/L, WM), all of liver Co values were below range, 14\% of liver $\mathrm{Cu}$ values were above range, all of kidney Se values were within range and all of liver Se values were above range. All of the herds had normal dam blood selenium, copper and magnesium status but only one had a normal blood iodine status; the others were high. Based on the clinical histories and necropsy and laboratory findings, causes of perinatal mortality were classified into foetal disorders [17, (43\%); lethal congenital defects (5), umbilical haemorrhage (3), dead in utero (3), anoxia/eutocia (2), prematurity (2), goiter (1) and anaemia (1)], calving problems [14, (35\%); malpresentation (7), dystocia (3), uterine torsion (2), milk fever (1), twinning (1)], placental problems [5, (13\%), premature placental separation] and unexplained perinatal mortality [4, (10\%)].

Conclusions From this preliminary study it is evident that the majority of perinatal mortality cases were stillborn, singleton males from pluriparae sired by Holstein-Friesian bulls with partially or completely atelectatic lungs without significant infections or micronutrient imbalances; calf mortality was primarily associated with foetal disorders and calving problems. The value of combining whole herd active surveillance with anamnesis and necropsy examination supported by laboratory testing is evident from the inclusion of both clinical and pathological diagnoses in the causes of death detected.

Acknowledgements The author acknowledges the technical assistance of Tom Condon and Jonathon Kenneally and the collaboration of colleagues in the Veterinary Laboratory Service of DAFF, AFBI and SAC.

\section{References}

Mee, J.F., Berry, D.P. and Cromie, A.R. 2008. Animal 2, 613-620.

Mee, J.F. 2009. In: Animal Reproduction: New Research Developments, Ed. L.T. Dahnof, Nova Science Publishers, Inc, 67-106. 